\title{
Fundamentos de la acción diplomática corporativa en las estrategias de internacionalización de empresas
}

Foundations of Corporate Diplomacy in Internationalization Strategies

RECIBIDO: 28 DE NOVIEMBRE DE 2021/ACEPTADO: 27 DE ENERO DE 2022

\section{MILAGROS DE TORRES FERNÁNDEZ \\ Instituto de Comercio Exterior miladetorres@telefonica.net ORCID: 0000-0001-9922-5426}

Resumen: Un nuevo concepto se ha abierto paso en la acción exterior de las empresas: la diplomacia corporativa, recurso de buenas prácticas que proporciona a las compañías fórmulas de eficacia probada para moverse en un mundo de escenarios complejos y cambiantes.

El estudio de los precedentes históricos demuestra que es perfectamente aplicable a la acción corporativa internacional. Estas técnicas, basadas en los principios tradicionales de la diplomacia pública, permitirán a las empresas desarrollar acciones que trasciendan lo puramente comercial para establecer vínculos humanos y económicos duraderos; estrategias que humanicen el cometido empresarial como procedimiento idóneo para la expansión comercial de nuestros días. Con ese objetivo, el diplomático corporativo debe mostrar determinados saberes técnicos, habilidades profesionales y cualidades humanas para lograr ejercer influencia, descubrir ámbitos de cooperación en el país meta y cimentar la buena reputación de su empresa, es decir, todas las áreas en las que resulta eficaz la diplomacia corporativa. Es el caso de compañías que han constatado la rentabilidad de la diplomacia empresarial con éxitos de gestión y reconocimiento internacional

Palabras clave: Diplomacia, Diplomacia Corporativa, Negociación, Reputación, Entendimiento Cultural

\author{
MARÍA TERESA Mula SÁNCHEZ \\ Universidad Internacional de Valencia \\ maytedoctorado@gmail.com \\ ORCID: 0000-0002-6451-7530
}

\begin{abstract}
A new concept has made its way into the external action of companies: corporate diplomacy, a resource of good practices that provides companies with proven formulas to navigate in a world of complex and changing scenarios.
\end{abstract}

The study of historical precedents shows that corporate diplomacy is perfectly applicable to international corporate action. These techniques, based on the traditional principles of public diplomacy, will allow companies to develop actions that go beyond the purely commercial aspects to establish lasting human and economic links; strategies that humanize the business mission as an ideal procedure for the commercial expansion of our days. With this objective, the corporate diplomat must show certain technical knowledge, professional skills and human qualities to be able to exert influence, discover areas of cooperation in the target country and build the good reputation of their company, that is, all the areas in which corporate diplomacy is effective. This is the case of companies that have seen the profitability of business diplomacy reflected in management success and international recognition.

Keywords: Diplomacy, Corporate Diplomacy, Negotiation, Corporate Reputation, Cultural Understanding 
La paz y la seguridad necesitan la cooperación política y económica de los estados Carta de Naciones Unidas

\section{INTRODUCCIÓN}

En la intrincada red de relaciones internacionales que dibuja el mundo actual, todo procedimiento que se encamine a lograr entendimiento y aproximación debe verse como una forma aplicable a cualquier ámbito en el que interese acercar posturas, ya se trate de estados, organizaciones supranacionales, empresas o personas. Con ese propósito, desde hace pocos años, ha cristalizado una expresión que recoge con eficacia esta nueva visión del papel de las empresas y corporaciones en su relación $-\mathrm{y}$ su compromiso social y productivo- con el mundo: la diplomacia corporativa. Del interés que suscita el concepto y su área de influencia habla la nutrida nómina de autores que lo abordan. Cabarcos y Ponz ${ }^{1}$ mencionan en su estudio sobre la diplomacia corporativa como herramienta de gestión, «tanto en el mundo académico como en el profesional», 19 definiciones relevantes del término.

Para centrar los ejes de esta exposición, partiremos de la que proporciona Manfredi ${ }^{2}$. En el capítulo II, tras recoger las teorías más destacadas con el propósito de delimitar el alcance del concepto y su ámbito de acción, llega a una conclusión: el término «ofrece una definición fluvial», lo que se antoja bastante abierto, pero inmediatamente acota algo más el campo:

Se concluye que la diplomacia corporativa es el desarrollo instrumental de la estrategia que ordena las relaciones con los poderes públicos y privados. Se concreta en el establecimiento de relaciones con terceros — con capacidad de acción o toma de decisiones - con el ánimo de resolver problemas propios y ajenos, la creación de marcos comunes de trabajo y la generación de confianza.

En ello se advierten dos concepciones: la primera alude a la visión tradicional que considera la acción diplomática una competencia exclusiva de los estados; la segunda, y más próxima al objetivo de este artículo, refiere la toma de conciencia de las organizaciones empresariales sobre el extenso campo que deja al descubierto la gran política exterior de Estado y que muy bien pueden

\footnotetext{
1 Cabarcos, R. y S. Ponz, C. (2017).
}

2 Manfredi, J. L. (2018), p. 54. 
abordar las empresas. En este sentido, la formulación de A. Camuñas ${ }^{3}$ es esclarecedora:

La diplomacia corporativa nace para cubrir las necesidades de unas empresas cuyo papel ha cambiado muy notablemente en las dos últimas décadas, en las que las corporaciones han tomado conciencia de su mayor autonomía frente a los poderes públicos y de las carencias de estos últimos para contribuir rápida y diligentemente en la resolución de sus conflictos y crisis en otros mercados siguiendo el patrón propio de la diplomacia internacional, de los servicios diplomáticos nacionales

Pueden incluso encargarse, con inmejorables perspectivas de éxito, de lo que es propio de su cometido: la expansión de las relaciones comerciales y la conexión civilizadora que ha supuesto el comercio desde tiempos remotos como fortalecedor de relaciones pacíficas y atenuador de conflictos. A ese respecto, vienen a la memoria las lúcidas palabras del economista, político y legislador francés de corte liberal, Frédéric Bastiat (s. XIX), firme defensor del libre comercio, cuando sostenía que «si los bienes no cruzan las fronteras, lo harán los soldados» ${ }^{4}$; o las de Kant, quien en su célebre obra La paz perpetua ${ }^{5}$ considera que el espíritu comercial no puede coexistir con la guerra.

Pues bien, ese espíritu de discreción y empatía, de búsqueda de afinidades, de creación de relaciones humanas de buena voluntad, de entendimiento cultural, de interés y respeto por las costumbres peculiares, previo o simultáneo a cualquier acción empresarial, es lo que Earley y Ang ${ }^{6}$ consideran y acuñan como «inteligencia cultural» (CQ), que alude a la «capacidad de integrarse y funcionar con efectividad en ámbitos pluriculturales». El artículo, por tanto, abordará la forma de asimilar los valores tradicionales de la diplomacia discreción, comprensión de los intereses ajenos y búsqueda del beneficio compartido, información, negociación e influencia y la humanización del cometido empresarial como procedimiento idóneo y más adecuado a la expansión comercial de nuestros días. Esa visión de mayor hondura humana de las empresas, tanto en su conciencia de responsabilidad social corporativa (RSC), como en su papel más humano y diplomático en el exterior, es lo que busca la diplomacia empresarial en su relación con los grupos de interés.

\footnotetext{
3 Camuñas, A. (2012).

4 Estas palabras se atribuyen con frecuencia a Bastiat (1801-1850), enérgico defensor del liberalismo, junto con Say, como forma menos impositiva y estatalizada, y más abierta, de la gestión económica.

5 https://biblioteca.org.ar/libros/89929.pdf.

6 Earley, P. C. y Ang, S. (2003), p. 12.
} 


\section{OBJETIVOS Y METODOLOGÍA}

Las líneas conceptuales mencionadas trazan el camino del presente artículo, que parte de una primera hipótesis respecto a que la diplomacia corporativa (DC) es un concepto actual que bebe, sin embargo, de fuentes históricas; y una segunda hipótesis que considera la DC como una estrategia de enorme valor añadido en la proyección internacional de las empresas.

Se trata, por consiguiente, de investigar, desde el punto de vista teórico histórico, descriptivo y analítico en primer término, de qué modo la diplomacia tradicional sirve de modelo a la corporativa y, también, en qué medida la diplomacia empresarial no es un concepto nuevo en sentido estricto, sino que históricamente se ha desarrollado en paralelo como complemento de la pública. El segundo objetivo es discernir si ambas formas establecidas de diplomacia pública y privada o extraoficial pueden servir de pauta a la actual acción diplomática de las empresas, tanto en sus principios, como en sus procedimientos y, sobre todo, en los rasgos y capacidades que deben desarrollar los directivos que se dediquen a esta noble tarea en representación de sus corporaciones. Se cierra este estudio con un último objetivo: verificar si las empresas que incorporan entre sus acciones estratégicas la diplomacia corporativa obtienen resultados satisfactorios gracias a ella.

En primer lugar, por tanto, se establecerá su basamento histórico: el concepto de diplomacia oficial o pública, la acuñación del término y su consolidación teórica en las décadas del prerrenacimiento y el siglo XVI; a continuación, se observará el desarrollo progresivo de esa diplomacia informal que empresas, compañías y personalidades de la vida social, la cultura y el comercio llevaban a cabo históricamente con diversos fines, ya fueran públicos, al servicio de sus gobiernos y cortes, ya privados, en beneficio de acuerdos corporativos.

En segundo lugar, se abordará su adaptación a los ámbitos de acción de la empresa en sus aspiraciones de internacionalización, tomando como referencia los acuerdos y convenios diplomáticos que definen las funciones de legaciones y embajadas. Se estudiarán aquí dos aspectos: en el primero, se definirán los atributos idóneos de los directivos empresariales que se dediquen a la representación corporativa no estrictamente comercial, sino en el ámbito de la imagen, la cooperación y las relaciones humanas que aseguran el entendimiento y la colaboración; el segundo se centrará en los campos de acción de las empresas. 
Se propondrá, por último, el caso de una compañía que ya ha aplicado esa acción empresarial que pone en valor el capital relacional como forma de compromiso con el avance de la sociedad. El estudio de caso responde a una metodología de investigación cualitativa que permite avanzar en el conocimiento de determinados fenómenos corporativos como es el que las empresas conviertan sus áreas de proyección internacional en centros de estrategia y desarrollo de relaciones en clave diplomática ${ }^{7}$. En concreto, el estudio descriptivo y explicativo propuesto se centra en la empresa Iberdrola, compañía con un elevada y reconocida proyección internacional que en cinco años ha pasado a desarrollar las dos terceras partes del negocio fuera de España, lo que - en palabras del asesor del presidente de Iberdrola — les exigió «tener un enfoque de diplomacia corporativa» $\mathrm{y}$ «asumir ese concepto de estrategia» para consolidar sus procesos de internacionalización en diferentes países. Por lo tanto, cabe considerar la unidad de análisis o estudio de caso único (Iberdrola), aunque suficientemente esclarecedora para estudiar los resultados de su aplicación, siendo conscientes de que cuanto mayor fuera el número de casos investigados la extracción de conclusiones sería más demostrativa; no obstante, se ha tratado de seleccionar un caso que ofrece mayor oportunidad de aprendizaje.

De todo el conjunto, se extraerán conclusiones en las que se valore este procedimiento renovado de acción corporativa, encaminado a dar solidez a las empresas en su quehacer productivo, sin descuidar los aspectos más humanos y valiosos de la relación; es decir: qué ventajas proporciona la proyección diplomática corporativa que se propone ir más allá de la simple acción industrial, comercial o administrativa y no solo busca el beneficio material.

\section{BREVE APROXIMACIÓN A LA BASE HISTÓRICA}

\section{El camino de la diplomacia pública.}

El vocablo «diplomacia» entró en el español por vía francesa (diplomatie) pero, como tantos otros de uso especializado, procede del mundo clásico: llega de Roma (diploma-atis, 'documento oficial') aunque su cuna está en Grecia $(\delta \imath \pi \lambda \tilde{v} v)$ — se pone de manifiesto continuamente lo que sostenía Gombrich ${ }^{8}$

\footnotetext{
7 Barciela, F (2010)

8 Gombrich, E.H. (2014), p. 51.
} 
respecto a que «todos nosotros somos alumnos de los griegos»- El primer registro data de $1823^{9}$, el poeta Quintana lo emplea en una de sus Cartas a Lord Holland $^{10}$; el uso referido a los 'tratados entre estados' debió de asentarse con rapidez porque la Academia ya lo recoge en 1832. El término, desde su origen griego ('tablilla o papel doblado' en dos), presenta ese concepto dual: en primera acepción, el 'documento oficial', del que deriva el carácter público y el valor de hecho registrado en un documento; en segunda acepción, lo relativo a la 'doblez', que puede entenderse como lo reservado y secreto de las decisiones, conversaciones o acuerdos, propio de la actividad que se desarrolla de forma discreta y sigilosa, que cuadra adecuadamente con la reserva de lo que se trata entre estados o gobiernos, pero también con cualquier conversación formal que requiera guardar discreción y prudencia en el contexto de los negocios.

«La diplomacia, entendida como negociación entre dos o más partes poseedoras de poder político, remonta su origen hasta prácticamente los primeros años de existencia de los reinos e imperios más antiguos ${ }^{11}$. Heródoto, en su Historia, acuña ya términos ligados al lenguaje diplomático, así, se refiere a «embajadores» y «delegación» en los libros III $(58,1)$ y V de $(93,1)$ entre otros; expresiones que aprovecha también Tucídides en el libro I de las Guerras del Peloponeso, a las que añade «enviados» $\mathrm{y}$ «emisarios», vocablos que insisten en la labor de representación mediadora en conversaciones de paz. Julio César emplea a menudo en La guerra de las Galias, la voz «legati» (legados). La Edad Media añade «heraldo» 0 «faraute» ${ }^{12}$, tomado del francés béraut, cuyo significado entronca más con la mediación, la transmisión de un mensaje y la representación oficial del príncipe: se espera de ellos capacidad para hablar persuasivamente y defender con fidelidad el pensamiento y los intereses de quienes lo envían. Alonso de Palencia (s. XV) incluye en una de sus obras, La batalla campal de perros contra lobos ${ }^{13}$ alegoría de corte político sobre las guerras nobiliarias durante el reinado de Juan II de Castilla , la figura del

9 Banco de datos (CORDE) [en línea]. Corpus diacrónico del español. <http://www.rae.es>

10 Quintana, M.J. [1853 (2010)], p. 22.

11 López Amores, A. (2014).

12 Nebrija usa la expresión «faraute de lenguas» como 'intérprete' o 'traductor'; de hecho, es la primera palabra que alude a quienes se aplican a este oficio; y la Academia aún lo recoge como una de las acepciones de «heraldo».

13 Martín Romero, J. J. (2016) y Maíllo-Pozo, R. (2014). 
faraute: la raposa Calidina $^{14}$, que actúa como mediadora entre ambos bandos contendientes. También en el XV y en Venecia, se reglamentan las funciones diplomáticas ${ }^{15}$ y más tarde se consolida «embajador»; pero también vocablos como «nuncio» $\mathrm{y}$ «legado» se aplican como sinónimos. Lo cierto es que en esos siglos «las empresas diplomáticas tenían una misión claramente definida que debía lograrse, tales como la firma de un tratado de paz, una alianza, acuerdos de no agresión, enlaces comerciales [...] Apenas la tarea encomendada había sido cumplida o fracasada, la comitiva enviada para realizar las negociaciones debía regresar» ${ }^{16}$. A este respecto, los actuales esfuerzos de la diplomacia corporativa se asemejan, por un lado, a la definición de propósito de la prerrenacentista, pero, por otro, estas nuevas fórmulas de delegados empresariales se proponen cimentar a largo plazo los vínculos establecidos, de modo que su labor se plantea con más alcance y permanencia.

La Edad Moderna, con el complicado sistema de relaciones entre estados, países nuevos y fronteras vacilantes, además del fermento de la primera globalización económica con el descubrimiento de América y la incorporación de nuevos productos al mercado, impulsa el verdadero despliegue de la diplomacia — «giro copernicano» lo considera López Amores—, gracias a grandes tratadistas, por un lado, y a gobernantes que robustecen su poder con embajadas permanentes, por el otro. Todo ello consolida a los embajadores, como ya se les conoce, y beneficia a sus representados porque «proporcionaban un flujo de información constante, tanto de mensajes oficiales como de rumorología; garantizaban, además, un canal de negociación abierto en todo momento, incluso en las peores situaciones, cuando más necesaria era la diplomacia; y permitían ampliar la red de influencia y control, favoreciendo el entramado de relaciones» ${ }^{17}$. Los estudiosos ${ }^{18}$ coinciden en que fue Fernando el Católico con su intensa e infatigable labor internacional, el primero en establecer una entrenada red de «procuradores» permanentes que permitiría moverse con seguridad por el espinoso escenario político del momento.

Respecto a los teóricos, tres nombres destacan: Rosier, Castiglione y De Vera. Del primero, cuya obra Breve Tratado de los Embajadores fue terminada

14 La exégesis literaria considera que tras este personaje se esconde la reina de Aragón, Juana Enríquez, hija del almirante de Castilla y madre de Fernando el Católico, por su papel en el propósito de conciliar al príncipe Carlos con su padre, Juan II, rey de Navarra y Aragón.

15 Fernández Carrasco, E. (2012).

16 López Amores, A. (2014).

17 Ochoa Brun, M.A. (2002), p. 75. 
en la corte de Castilla en 1436, se extrae la recomendación humanista de la búsqueda de la paz en la tarea de los embajadores; Mattingly destaca el carácter ético y conciliador que debe perseguir cualquier acción diplomática, ya sea política o comercial ${ }^{19}$. Respecto a Castiglione - exquisito cortesano, formado en las más prestigiosas cortes de la época, Milán, Urbino, la sede pontificia y la nunciatura como cardenal en la corte del emperador Carlos V-, en su obra El cortesano define «el excelso "arte de la cortesanía", esto es, el código de comportamiento, el conjunto de valores y reglas en los que se fundaba una "forma de vida" — distinguida, elegante y decorosa- propiamente cortesana $»^{20}$, característico de las nuevas necesidades y funciones de aquella corte de compleja proyección internacional, urbana y civilizada, que estimulaba entre cortesanos, más aún si lo eran de oficio, los "valores puros" y los "ideales nobles": el concurso de las exquisitas maneras, fruto de la buena crianza y, desde luego, los saberes humanísticos del nuevo siglo, el dominio del lenguas, el conocimiento de artes, el buen gusto y el trato refinado pero natural que huye de lo exagerado y artificioso... En lo esencial, estas recomendaciones no han envejecido y pueden servirnos hoy como base para dibujar los rasgos esperables en quienes ejerzan el sutil quehacer de defender los intereses corporativos con miras más elevadas que el rápido beneficio comercial. La tercera de estas figuras es la de Juan Antonio de Vera, el primer europeo en elaborar un volumen coherente sobre la diplomacia y todos sus aspectos internos, que escribe El Enbaxador en 1620, obra difundida rápidamente por Europa gracias a que glosa con agudeza el papel de los embajadores y los atributos que deben reu$\operatorname{nir}^{21}$; Vera pretendía no solo «explicar lo que era un embajador, sino cómo había de ser el que aspirase a la perfección en el oficio $\gg^{22}$. Ya se ve en su escrito el cambio de paradigma: del humanismo de inspiración clásica, a la separación, iniciada por Maquiavelo, entre «razón de Estado» y moral.

\section{Hacia la diplomacia informal.}

Aunque establecida a partir de finales del XVII — con frecuencia se señala la Paz de Westfalia como arranque del proceso- la que se podría llamar diplomacia residente u oficial, es decir, la función diplomática ejercida por un

\footnotetext{
18 Ballesteros García, A. (1995), pp. 50-51 citado en López Amores, A. (2014)

19 Mattingly, G. (1970), pp. 148-149.

20 Torres Corominas, E. (2010).

21 López Amores, A. (2014).

22 Ochoa Brun, M. A. (2002), p. 244.
} 
representante permanente, con un estatus definido y un carácter funcionarial, la diplomacia sottovoce no pierde vitalidad. A menudo los gobernantes recurrieron a figuras particulares, casi siempre autorizadas por su relevancia y su facilidad para moverse en la corte y acceder a los círculos más restringidos del poder, o con prestigio cultural (músicos, escritores, pintores, banqueros, empresarios...). Eran lo que podíamos llamar «embajadores informales», que recibían reservadamente un encargo peculiar, en ocasiones asociado a los prolegómenos de una negociación de acuerdos comerciales o estratégicos en la que había de tejerse un delicado entramado de intereses que convenía conocer, o para informarse de particularidades culturales que podrían entorpecer las conversaciones si no se entendían y se respetaban; a veces los encargos iban ligados a la proyección de una imagen favorable del país enviante; o se proponían lograr una actitud receptiva para determinadas medidas que no serían bien recibidas sin su eficaz persuasión. «Todos ellos contribuyeron a construir las relaciones internaciones entre distintas cortes y con estrategias distintas, allí donde no llegaba el control de los soberanos ni de sus consejos de gobierno ${ }^{23}$; la misma autora sostiene que se encomienda a comerciantes, mercaderes viajeros, religiosos, etc. la entrega de mensajes y de regalos de buena voluntad, faceta muy a propósito de lo concerniente a la diplomacia corporativa. Una nueva cultura diplomática asociada al «buen gusto y a la figura del amateur y del diletante».

Entre las figuras de artistas prestigiosos que recibieron encargos diplomáticos de sus respectivos soberanos o de gobiernos aliados, destacan, por su relieve e influencia, Rubens y, en menor medida, Velázquez. El gran pintor flamenco prestó valiosos servicios a la monarquía española para lograr superar los desencuentros entre Londres y Madrid; sus buenos oficios culminaron en la firma del Tratado de Madrid (1630). Rubens deleitaba con sus modales exquisitos, su rica cultura clásica, habilidad oratoria, don de gentes y dominio de idiomas; su trato galante y su elegancia personal le permitían frecuentar los salones cortesanos y la proximidad a los personajes reales, que valoraban la grandeza de sus obras y la refinada sutileza de sus retratos: gran pintor y hábil cortesano que consiguió notables éxitos diplomáticos. Velázquez, aunque en un orden menor, logró, con sus eficaces recursos de negociador flemático y diplomático informal, la adquisición de un destacadísimo repertorio de obras de arte para las colecciones reales —más adelante, base del Museo del Prado, considerada la mejor pinacoteca del mundo-; la contratación de artistas y ar-

23 Carrió-Invernizzi, D. (2015). 
tesanos destacados para la corte, además de la apertura o la consolidación de relaciones con las familias más poderosas de Italia, en cuyas mansiones se alojó, y, por último, la benevolencia del papa, cuyo retrato pinta con asombrosa maestría, lo que supuso influencia favorable a los negocios de España.

Esto significa que, a lo largo de los siglos, han convivido las formas establecidas de la diplomacia oficial y las relaciones con propósito diplomático, menos institucionalizadas pero muy eficaces por ser más flexibles y adaptables al propósito. Todo ello, sin requerir grandes inversiones ni demasiado aparato para lograr su cometido, lo que a menudo era una ventaja porque permitía que su objetivo pasara desapercibido si era necesario.

\section{Formas diversas de la diplomacia no oficial: de los pactos entre comerciantes a la diplomacia corporativa.}

Las que mejor percibieron las ventajas de la diplomacia comercial fueron las compañías mercantes, necesitadas de vínculos amistosos y sedes permanentes que no estuvieran expuestas a los avatares de los cambios de gobierno. Por su propia iniciativa, ciudades y gremios establecían pactos y fraguaban alianzas con los de otras ciudades para regular las actividades comerciales, aproximar intereses y evitar competidores. Es el caso, en el norte de Europa, de la Liga Hanseática cuya labor mercantil, pero también de relaciones diplomáticas hábiles, logra a finales del XIV un poderío comercial y un grado de bienestar para sus ciudadanos que hablan de los beneficios de tales relaciones. Similar es el caso de las compañías de las Indias que alcanzaron provechosos acuerdos con los próceres de los países de destino y una red comercial que beneficiaba no solo a los propios mercaderes sino al país de origen, del que perfilaban una imagen de prosperidad y progreso que constituía un modelo. A esas compañías siempre podían recurrir los gobernantes para conseguir propósitos de otra índole valiéndose de la fluidez y llaneza de sus relaciones con los naturales, de manera que, entre ambas formas, la pública y la privada, se ha producido tradicionalmente un verdadero trasiego de relaciones; de hecho, las compañías comerciales reproducían los modelos de la diplomacia oficial con normas, convenios y relaciones estables encaminadas a un objetivo. Lo que realmente las diferenciaba es que quienes la ejercían no eran funcionarios públicos — legados, cónsules, embajadores, nuncios, etc.—, sino agentes privados: comerciantes, marinos, mercaderes, comisionistas, intermediarios... Tal acción se acerca ya al concepto de nuestro interés, la diplomacia corporativa.

No obstante, conviene, antes de abordarla plenamente, detenerse en otro concepto afín que tiene, sin embargo, un campo conceptual específico; se trata 
de la diplomacia comercial. Enrique Fanjul se teme que «existe, en primer lugar, una cierta imprecisión sobre el significado del término "diplomacia comercial" $\gg^{24}$ y se propone esclarecer el concepto:

La diplomacia comercial debe ser entendida como un canal para la ejecución de las políticas de internacionalización de la economía y la empresa. Es decir, se puede definir como la política de internacionalización de la economía y la empresa que se ejecuta a través de las representaciones diplomáticas de un país. Como la define Olivier Naray, "la diplomacia comercial es una actividad desarrollada por representantes de un Estado con status diplomático con el objetivo de promover los negocios entre su país y el país el que se encuentran destinados”.

El autor deja claro que se trata de una acción diplomática pública, inspirada por la propia autoridad política, que responde a una estrategia de Estado y se ejecuta a través de las embajadas a cargo de funcionarios del cuerpo diplomático que «suelen tener el título de consejero o agregado comercial». Destaca las siguientes líneas de actuación: promoción comercial de los productos y la imagen del país; apoyo financiero público a la internacionalización; capacidad de influencia política, militar o económica para defender sus intereses en los mercados internacionales; servicios de apoyo a las actividades internacionales de las empresas; participación en el marco institucional del comercio internacional; y subvenciones y desgravaciones fiscales a la exportación e inversiones en el exterior.

Ciertamente, la diplomacia comercial realiza una serie de funciones medulares en el apoyo a las empresas en su propósito de expansión internacional; a este respecto, los Servicios Personalizados de $\operatorname{Ice}^{25}$ llevados a cabo por la red de ofecomes, ofrecen múltiples servicios cuyo objetivo no es diplomático, sino de información económica, social, política y estructural del país de destino. Entre sus actividades, Fanjul destaca estas:

1. Búsqueda de potenciales socios y clientes

2. Networking o redes de contactos

3. Información o inteligencia económica

24 Fanjul, E. (2013).

25 Instituto de Comercio Exterior (ICEX España Exportación e Inversiones) que gestiona una red de Oficinas Económico Comerciales de las Embajadas de España en el extranjero al servicio de la internacionalización de las empresas españolas. 


\section{Promoción comercial y de la imagen del país}

\section{Apoyo político}

El esclarecedor artículo no deja lugar a duda sobre que se trata de una forma específicamente económica de la diplomacia oficial, con la que puede colaborar estrechamente en algún caso la acción de la diplomacia privada de las empresas y corporaciones, pero no puede confundirse con ella. No obstante, aunque queden bien delimitadas las áreas de ambas formas de acción diplomática, autores como Mogensen, citado por Bolewski ${ }^{26}$, encuentran en la diplomacia corporativa una herramienta prometedora, un «diamante», para la diplomacia comercial. Es decir, una forma de lograr legitimidad y solvencia ética en las relaciones puramente comerciales; o dicho de otro modo: estimular a las empresas a emprender acciones en ambas vías diplomáticas la corporativa y la comercial para fidelizar a un cliente que muestra claras preferencias hacia «una economía solidaria centrada en el ser humano» de «sostenibilidad ambiental y justicia social».

\section{VISIÓN ACTUAL DE LA ACCIÓN DIPLOMÁTICA DE LAS EMPRESAS: LA DIPLOMACIA CORPORATIVA. FUNCIONES Y ACTIVIDADES.}

En un mundo de fronteras permeables para el comercio y confluencia casi infinita de productos, a lo que se suma el mercado abstracto que abre internet con su inagotable oferta, los papeles de los consumidores y de los productores han cambiado drásticamente. El cliente actual, más informado, crítico y asediado por la oferta que nunca, no compra productos sino expectativas, coincidencias ideológicas o emotivas y, en consecuencia, rechaza los que no le resulten afines pues el mercado ofrece otros que satisfacen sus intereses sin contrariar sus principios.

El mercado, gracias a la globalización, es inmenso, así que la perspectiva internacional es horizonte obligado porque la competencia se ha hecho universal. Sin embargo, las empresas saben muy bien que, aunque el mercado sea global y los productos lleguen a cualquier rincón del planeta, han de mimetizarse con el entorno si se quiere que los naturales de esa región, grupo ideológico o espacio los acojan favorablemente; y saben también que su reputación y la de su marca están al albur de la opinión pública, más volátil e

26 Bolewski, W. (2021). 
inconsistente que nunca por la multiplicación de mensajes y opiniones. Por todo ello, el camino al consumidor no es un vector único, lo que implica muchas más líneas de acción y de áreas, es decir, trabajar con grupos de interés externos a la organización: agrupaciones de consumidores, líderes de opinión, medios de comunicación clásicos, sector financiero, grupos de distribución, sindicatos y círculos empresariales, organismos reguladores y, naturalmente, autoridades públicas de los diversos niveles de la administración; sin olvidar el «continente» de internet y las infinitas opiniones vertidas en las redes sociales, que dan voz a millones de usuarios anónimos y silenciosos hasta que, armados de sus dispositivos móviles, se imponen como medio de comunicación de masas y de expresión personal. Todo eso ha acarreado una hiperproducción de mensajes y la multiplicación de polos de información, la creación de noticias falsas o de relatos interesados que vilipendian un producto, una compañía o ensalzan fugazmente otro... De acuerdo con Joseph S. Nye, Jr., mencionado por Camuñas ${ }^{27}$, podemos decir que «en la era de la información, ganará el que mejor mensaje tenga».

Ese es el espacio en el que se abre paso el concepto de diplomacia corporativa, un recurso de buenas prácticas, «un poder blando para todos los actores diplomáticos no estatales (multinacionales, ONG e incluso ciudades inteligentes) $\gg^{28}$ una eficaz forma de representación empresarial en las relaciones multilaterales, que puede muy bien delimitarse conceptualmente con la definición de Saner, profesor de la universidad de Basilea, que recoge Asquer ${ }^{29}$ :

Incluye diversas actividades como la capacidad de influencia sobre diferentes actores para crear y explotar oportunidades de negocio, colaborar con autoridades públicas y en el proceso de toma de decisiones que afectan a los procesos comerciales y de inversión, así como, prevenir posibles conflictos con los grupos de interés externos y reducir los riesgos políticos, además de atraer a los medios y a los líderes de opinión para salvaguardar la imagen corporativa y la reputación de la empresa.

Para lograr influencia social, como propone el profesor suizo, las empresas necesitan que se les conozca, que se aprecien sus valores, «aquí es fundamental la dimensión humana, la capacidad de entender los asuntos en clave

27 Camuñas, A. (2012).

28 Sevin, E. (2021).

29 Asquer, A. (2012). 
personal $\gg^{30}$ y que se reconozca su contribución al bienestar y al progreso. Por tanto, se abre un campo inmenso para las relaciones humanas desarrolladas en el ámbito económico y productivo cuyo propósito es suscitar adhesión: «las empresas han de relacionarse y establecer un conjunto de prioridades que alineen el interés del ciudadano con los objetivos del negocio ${ }^{31}$, buscar la complicidad en valores compartidos (la protección del medio ambiente, de la infancia, de los derechos humanos...), hacer sugestiva la imagen de la marca, cimentar el buen nombre de la empresa, lograr el apoyo de un sector de prestigio y el marco de un país apreciado del que los consumidores tienen buena opinión. Es la relación no impositiva ni subordinada, sino basada en atributos blandos. A juicio de Egea Medrano ${ }^{32}$, «tener socios locales, preocuparse por la sociedad en la que se implantan, crear redes de información, de influencia con los grupos de interés clave del país anfitrión, son ejemplos de las herramientas que emplean las empresas multinacionales en sus interacciones y su estrategia de no mercado; en otras palabras, usan un enfoque relacional». En síntesis: buena calidad en los productos, excelencia en la imagen, sólida reputación; a este respecto, es muy cierta la idea de que «la imagen exterior de un país es un activo intangible que favorece la actividad económica» ${ }^{33}$. Y todo eso no puede hacerse sin una estrategia corporativa de relación diplomática con todos los que toman decisiones.

Llegados a este punto, cabe preguntarse cuáles son las funciones de la diplomacia corporativa y cómo aplicarla si se aspira a lograr lo propuesto más arriba. Para ello, vamos a tomar como referencia el artículo 3 de la Convención de Viena sobre Relaciones Diplomáticas (18 de abril de 1961), en el que se recogen las funciones de la misión diplomática de un Estado, y las aplicaremos, mutatis mutandis, a las que puede desarrollar la empresa en su estrategia diplomática. Vistas sintéticamente, las cinco funciones consisten en a) representar; b) proteger sus intereses; c) negociar; d) enterarse lícitamente de los acontecimientos e informar de ellos; y e) fomentar las relaciones amistosas y desarrollar las económicas, culturales y científicas.

Tales funciones pueden muy bien desplegarse en el ámbito corporativo mediante acciones que conviertan a la empresa en un ente visible, colaborador en proyectos compatibles con su cometido en el país receptor o con las

30 Manfredi, J. L. (2018), p. 48.

31 Manfredi, J. L. (2018), p. 33.

32 Egea Medrano, M. A. (2016).

33 Romero, I. y Rodríguez-Gutiérrez, M. ${ }^{\mathrm{a}}$ J. (2014). 
empresas extranjeras con las que trabaje. Con ello, sus intereses y su reputación corporativa estarán mejor protegidos que si no emprendieran acción alguna; de hecho, Edward Freeman ${ }^{34}$ sostiene que «existen numerosos estudios que indican que una buena reputación es rentable para los accionistas, lo que se ha convertido en la justificación económica de la gestión de la reputación». Veamos esas funciones diplomáticas aplicadas al ejercicio empresarial.

a) La representación, que suele asignarse a los llamados country manager y está muy atenta a las corrientes de opinión, a la cultura y los valores intrínsecos de la sociedad de acogida, implica proyectar ante ella la mejor imagen de la empresa. Esto llevará, entre otras acciones, a participar en eventos en los que la empresa adquirirá rostro humano a través de quienes la representen, para lo cual, habrán de desplegar habilidades sociales, conocer la etiqueta asociada a las costumbres del destino. En el ámbito de tales eventos, se tiene la oportunidad de entablar interesantes relaciones con otros empresarios, autoridades públicas y personas de relevancia social que ayudarán a aumentar la red de contactos y relaciones humanas y empresariales valiosas.

b) En segundo lugar, descuella la función de negociar. En una mesa de negociación se deciden aspectos medulares y se asegura el avance económico de la empresa. Realizar con pericia esas complejas acciones de comunicación proporciona incontables beneficios y, si se consigue el objetivo de que ganen todos, se asegura la buena opinión de la contraparte y la consolidación de relaciones comerciales productivas y redes profesionales receptivas.

c) Para ello, es necesario manejar los mejores datos, que permitan analizar situaciones y estar preparados para actuar: «la diploma la corporativa, en su faceta de 'business inteligence', es una vía eficaz para conseguir. información relevante para el negocio de forma anticipada» $»^{35}$; con lo cual se llega a la tercera función: reunir la mejor información, la más actual y atenta al contexto; eso significa mantener los canales activos y permeables a los mensajes de diversos emisores, el objetivo es trazar una estrategia fundamentada en la realidad que tenga posibilidades de éxito y, al mismo tiempo, sea capaz de anticiparse a los cambios y prevenir los conflictos. También esa función incluye el objetivo de descubrir nuevas oportunidades de negocio y la readaptación de los productos a los gustos y rasgos específicos de los consumidores, y a los cambios que experimente el mercado. De hecho, en el lábil contexto actual, para la em-

34 Freeman, R.E. [1984 (2010)], p. 7.

35 Cabarcos, R. y S. Ponz, C. (2017). 
presa es ineludible examinar la abrumadora cantidad de información diaria y filtrarla con criterios de calidad eficaces para interpretar con acierto las "señales" del entorno no comercial, así como conflictos potenciales, involucrarse con las partes interesadas externas y tratar de influir en ellas ${ }^{36}$.

d) La protección afecta a las personas, a los intereses y a los productos. En el caso de la diplomacia corporativa, lo que puede ser susceptible de salvaguardia es la reputación de la empresa y la protección de los empleados que trabajan, ya sean directivos expatriados o naturales del país; saber que se trabaja en un entorno grato y seguro, al amparo de medidas adecuadas y en un ambiente acogedor, contribuye a la buena imagen de la empresa y a la confianza «de largo recorrido», en palabras de Henry Kravis ${ }^{37}$ de grupos de interés como sindicatos, agrupaciones profesionales y círculos empresariales.

e) Por último, la creación de relaciones es seguramente la más visible y, al mismo tiempo, la que más se asocia al éxito corporativo y a la creación de buena reputación. Es el caso de que se consulte a directivos o empleados sobre aspectos científicos, jurídicos o de otra índole en los medios de comunicación o dentro de la comunidad científica; la participación en foros, congresos, ferias, actividades culturales, etc. y recibir solicitudes de colaboración o reconocimientos por el trabajo realizado es la mejor medida para saber que la empresa ha echado raíces en el lugar de implantación, que la opinión que suscita es favorable y goza de buena reputación y capacidad de influencia. Conviene, además, realizar acciones filantrópicas, de patrocinio y mecenazgo que demuestren compromiso y sentido de la responsabilidad, así como el propósito de devolver a la sociedad una parte de lo recibido de ella. Sabemos que una empresa no solo crea valor económico cuando da beneficios a los accionistas: «Si da una imagen de responsabilidad, se gana la confianza de sus stakeholders, lo que en última instancia mejorará su negocio» ${ }^{38}$. A juicio de Henisz $^{39}$, la empresa internacional debe infundir legitimidad a sus acciones si quiere aumentar su ventaja competitiva; esto le obliga a adquirir un nuevo papel institucional que le permita crear valor no solo a clientes, sino para toda la sociedad y a largo plazo; a ese respecto, es un firme defensor de la diplomacia corporativa porque $\ll$ crea valor empresarial real $»^{40}$.

36 Egea, M. A.; Parra-Meroño, M.C. y Wandosell, G. (2020).

37 Cofundador de la empresa de adquisiciones Kohlberg, Kravis Roberts and Co. (KKR).

38 Harris, Jared D. y Freeman, R. Edward (2008).

39 Henisz, W.J. (2016).

40 Henisz, W.J. (2014), p. 8 
Cuando haya que crear valor, hay que buscar a los mejores o capacitarlos para que lo sean. Podemos partir de la advertencia que hace Camuñas ${ }^{41}$ respecto a lo delicado y ambicioso de este trabajo: «Se trata de un servicio altamente exclusivo y especializado que exige un conocimiento detallado de disciplinas como el marketing, la comunicación, las relaciones públicas, el lobby, las relaciones políticas y empresariales nacionales e internacionales, y - por encima de todo- una intensa experiencia en operaciones y proyectos de envergadura al máximo nivel político, económico o social». Lo importante será la promoción de los productos, la comunicación estratégica y las relaciones interpersonales. Como guía sencilla para los candidatos a esa noble tarea, la empresa debe requerir saberes técnicos, habilidades profesionales y cualidades bumanas que conviene desgranar para captar o entrenar al adecuado.

a) Veamos qué se requiere respecto a los saberes técnicos: conocer a fondo el mercado de destino, sus peculiaridades económicas, sectores y productos, estructura empresarial, necesidades y recursos, los modelos financieros de su economía y cuanto pueda contribuir a encontrar oportunidades de negocio, que es objetivo primordial.

b) En cuanto a las habilidades profesionales, es imprescindible que se maneje con fluidez en idiomas internacionales y conozca suficientemente el idioma de interés; tenga habilidades negociadoras, sepa escuchar y argumentar con eficacia y transparencia, atienda con respeto las diferencias culturales; sepa hablar en público para intervenir en foros o entrevistas; tenga un comportamiento adecuado en los negocios, cuide su imagen personal y conozca los rudimentos del protocolo que rige en el país porque habrá de participar u organizar actos; tendrá que saber relacionarse con autoridades y representantes de grupos de interés, la comunidad científica, las ONG, etc.

c) Por último, las cualidades humanas que se han de buscar nos hablan de humanismo y sentido ético: empatía, discreción, refinamiento cultural, excelentes modales, capacidad de relación, exquisito respeto por los demás y sensibilidad para las costumbres y tradiciones, en suma, inteligencia cultural.

Todo ello constituye un elenco de atributos que asegura la capacidad de ejercer con éxito la diplomacia corporativa para ser el mejor «embajador» de la responsabilidad, el compromiso, la transparencia y la capacidad de hacer negocios con productividad y prestigio para la empresa. Conscientes de la nece-

41 Camuñas, A. (2012). 
sidad de formar a este nuevo cuerpo de expertos, algunas universidades y escuelas de negocios han abierto estudios de posgrado y másteres universitarios ${ }^{42}$ para ofrecer esa función a una sociedad empresarial que hasta ahora contrataba los servicios de exembajadores, funcionarios de embajada o dirigentes retirados de la actividad política, en razón de los contactos generados por su actividad y por su conocimiento.

La diplomacia corporativa resulta particularmente eficaz si se trata de crear relaciones en países y áreas de las que, en función del modelo de Edward T. Hall, se consideran de «alto contexto», como el caso de Asia oriental, Mundo árabe, la India o países de África, cuya cultura de negocios constituye un obstáculo para muchas empresas españolas porque en ellas, a las barreras clásicas de entrada, se les suma la preeminencia de barreras culturales y la dificultad de sortearlas si no se entienden las tradiciones y los vínculos emocionales o los rechazos que dificultan la relación comercial. A este respecto, han surgido consultoras especializadas que prestan servicios a las empresas.; incluso algunas grandes empresas ya han creado sus propios gabinetes de diplomacia de negocios generalmente en conexión con la alta dirección.

\section{C1. ampos de proyección de la diplomacia empresarial.}

Como se adelantaba en la Introducción de este artículo, Cabarcos y S. Ponz ${ }^{43}$ en su Informe analizaron exhaustivamente las definiciones más destacadas sobre el término y extrajeron $\ll 30$ conceptos clave relacionados con 25 áreas funcionales de la empresa» y lo plasmaron en la figura siguiente.

42 Hoy, los alumnos egresados del programa de becas de ICEX, impartido desde hace décadas por la fundación CECO (Centro de Estudios Económicos y Comerciales), que realizan sus prácticas en las ofecomes, reciben una formación muy a propósito, pues se encamina a conocer y practicar todas las áreas de la internacionalización de la empresa, con desarrollo de habilidades y capacidades de actividad diplomática corporativa. Además, realizan dos años de prácticas, primero en una ofecomes (Oficina Económico y Comercial de España), donde absorben toda la realidad socio económica y política del país para ofrecérsela, en forma de estudios e informes, a las empresas, y, a continuación, un segundo año en los departamentos de internacionalización de diferentes empresas españolas.

43 Cabarcos, R. y S. Ponz, C. (2017). 
Figura 1. Escala de conceptos mencionados en las definiciones de Diplomacia Corporativa

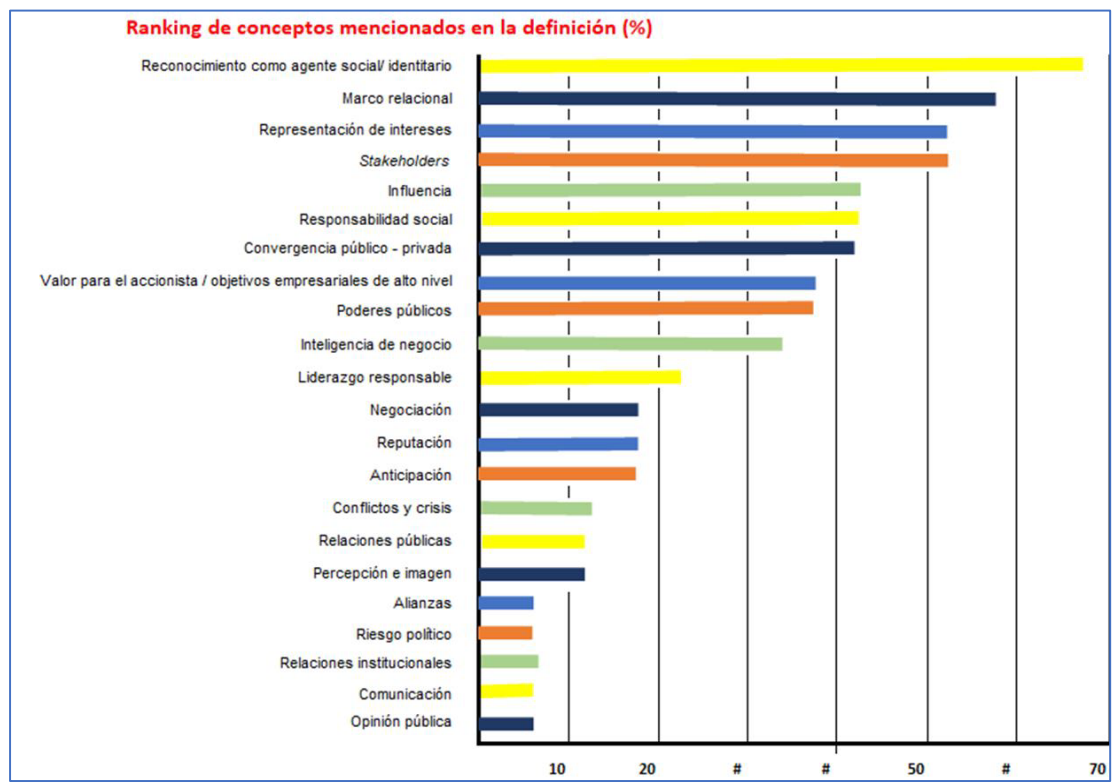

Fuente: Elaboración propia a partir del informe de Cabarcos, R. y S. Ponz, C. (2017)

De esta representación, como ellos mismos subrayan, sobresale un «fuerte componente identitario $(63,29 \%) »$; el «complejo marco de relaciones» $(57,9 \%)$ y el fundamento de la acción diplomática empresarial, que no es otra que la representación eficaz de los intereses y aspiraciones de la empresa ante los grupos de interés (stakebolders) en el ámbito internacional (52,69\%). De todo ello se desprende que la función más sobresaliente es proyectar con determinación la personalidad de la empresa, que se la conozca y se la valore para lograr influencia y prestigio; en segundo lugar, comprender la complejidad del tejido internacional en el que debe moverse para logar el tercer objetivo: identificar, conocer y apreciar a sus grupos de interés externos (organizaciones empresariales, clientes internacionales, organismos públicos, medios de comunicación, agentes sociales, competidores, comunidad científica, mercado y organismos reguladores, etc.) y reforzar a los públicos internos (accionistas, clientes, proveedores, socios, empleados, ámbito financiero, etc.).

Pero no es desdeñable todo el elenco de áreas e intereses que despliega la acción diplomática corporativa, según se desprende también del mencionado estudio de Cabarcos y Ponz. De forma que la acción diplomática empresarial, 
que ha de partir de la más alta dirección, es una estrategia a medio y largo plazo que debe centrarse, cuando se aspira a mantener relaciones empresariales sólidas en el mercado internacional, en ganar reputación, forjar alianzas productivas, entender el marco político del país o el área de destino, fomentar las relaciones institucionales, adelantarse a los problemas con la mejor información, neutralizar los conflictos y establecer vías de comunicación fluida con los grupos de interés. A este respecto, Mogensen ${ }^{44}$ reconoció los siguientes procedimientos de la diplomacia convencional perfectamente aplicables a la práctica corporativa: participar en diálogos con los públicos de las comunidades anfitrionas, para construir relaciones a largo plazo; compartir información; reconocer valores diferentes; mediar entre intereses distintos; moldear compromisos con los representantes de la sociedad civil. [...]; y armonizar intereses y expectativas divergentes dentro de un enfoque holístico de los aspectos emocionales, sociales ${ }^{45} \mathrm{y}$ de inteligencia intercultural.

En este nuevo universo de relaciones empresariales, la capacidad de expandirse económicamente no será ajena al desarrollo social y al sostenimiento de actitudes más humanas y dialogantes, como se exige hoy. Las empresas que quieran mantenerse con éxito en este intrincado tablero, habrán de hacerlo porque se aprecien sus productos, sí, pero sobre todo porque no se desconfía de su buen hacer, se reconoce su buena voluntad, su transparencia, su trabajo en beneficio de la concordia, su empatía cultural, y se aprecia su papel activo en la defensa de principios estimados por la sociedad de destino que toma decisiones de compra.

\section{EMPRESAS QUE APLICAN ACCIONES DE DIPLOMACIA CORPORATIVA}

En este nuevo marco de relaciones más líquido y competitivo, las empresas han asumido la importancia de ejercer un papel activo para consolidarse y crecer, pero también prestan más atención a la proyección de influencia, a difundir valores positivos asociados a su marca y a la humanización de esos vínculos con todos los grupos de interés. El resultado es que se ha abierto paso, en los consejos de administración y en los rangos más elevados de dirección, el concepto de diplomacia corporativa como estrategia de dirección. 
Las empresas como Iberdrola, Meliá, Inditex, Indra o Acciona han pasado de ser compañías con ADN español, a ser entidades globales con la mayor parte de su negocio fuera; Egea Medrano ${ }^{46}$ recoge en su tesis este ilustrador párrafo respecto a ese cambio radical de las empresas españolas, que en breves años han pasado del ámbito doméstico a la esfera internacional en los niveles más elevados y competitivos:

En 1985 nadie hubiera imaginado que un grupo textil español (Inditex) conquistaría el mundo con 6.777 tiendas en 88 países; [...] que una entidad financiera española (Banco Santander) sería el primer banco del área euro por capitalización; [...] que una constructora española (ACS) liderase el listado de los grupos de construcción e ingeniería con mayor negocio internacional del planeta... (David Fernández. El País 15-10-2016)

Esto les ha impulsado a considerar estrategias cuyo enfoque toma en consideración la diplomacia corporativa, más aún en empresas expuestas a sectores regulados como la energía, la banca o las concesiones de transporte que, además, las sobreexponen públicamente. En este caso, el principal enfoque de su acción diplomática corporativa tendrá como objetivo generar confianza basada en el compromiso; dicho de otro modo, han tomado conciencia de la necesidad de esforzarse en ganar la confianza de los ciudadanos para lograr buena reputación. En tales circunstancias, la responsabilidad social corporativa (RSC) se convierte en el eje de esta nueva diplomacia.

Desde hace algunos años, empresas de proyección internacional reconocida, como Iberdrola, Santander, Telefónica, Repsol, BBVA o la compañía hotelera Meliá cuentan con gabinetes de profesionales dedicados a ejercer la diplomacia corporativa. En los dos últimos años la inefable pandemia de la COVID-19, que ha supuesto un cataclismo para la marcha de la economía, ha promovido, sin embargo, encomiables acciones de solidaridad corporativa; es el caso de Zara al brindar su red logística y sus relaciones con mercados de abastecimiento para proveer rápidamente de materia sanitario imprescindible. Son formas nuevas de cooperación que «demuestran la urgencia de establecer alianzas público-privadas, entre el gobierno y los negocios, centradas en el ser humano; proporcionar servicios financieros, económicos y fórmulas de superación en situaciones de catástrofes internacionales. El gobierno se beneficiará de la experiencia corporativa en la gestión de riesgos [...] y las empre-

46 Egea Medrano, M. A. (2016). 
sas apreciarán la cobertura del gobierno en intervención y seguridad $\gg^{47}$. En este contexto internacional, menos previsible y más volátil, afectado por nuevas realidades y entornos geopolíticos ${ }^{48}$, las empresas multinacionales y sus directores ejecutivos han entrado en «la arena diplomática» ${ }^{49}$.

Para nuestro análisis, se ha seleccionado como ejemplo de esta línea estratégica la empresa Iberdrola, cuya labor en esta área ha sido galardonada en 2017 con el Premio al Mejor Gobierno Corporativo por la revista World Finance. A juicio de su presidente, el modelo de negocio de la empresa está «basado en la ética y la transparencia, la visión a largo plazo y la integración de culturas y personas» ... Ya en ese año, Iberdrola contaba con una amplia presencia en América Latina gracias a proyectos que, en México, por ejemplo, la consolidan como el mayor productor de electricidad privado del país.

La compañía desarrolla una firme estrategia de compromiso social con las comunidades en las que se integra; en consecuencia, lleva a cabo acciones de protección y ayuda, como la reciente donación en noviembre de 2020 para apoyar a familias afectadas por las inundaciones en los estados de Chiapas y Tabasco. El acto en el que el CEO de Iberdrola México y el presidente de la Confederación de Cámaras Industriales (Concamin) hicieron la entrega simbólica de la donación a los senadores de Chiapas y Tabasco trata de conjugar responsabilidad y compromiso con labores de representación y relación que favorecen la reputación de la marca y la mejora sus relaciones institucionales.

Otro de sus más decididos compromisos es la lucha contra el cambio climático; en concreto, reducir un 50 por ciento la intensidad de emisiones de $\mathrm{CO}^{2}$ en 2030 y ser neutra en emisiones de carbono en 2050. Compromisos que se traducen en actividades que suponen cuidar las relaciones y el entorno, y mostrar una actitud de preocupación por el medio ambiente. De esta forma, lleva a cabo un taller sobre el cambio climático en el que implica a 33 escuelas participantes comunidades en su mayoría en las que Iberdrola México mantiene instalaciones productivas . De esta forma, la empresa enfoca sus relaciones más allá de lo puramente económico, y en su actividad institucional no comercial, en la que involucra todos los grupos, crea valor sostenible y promueve el desarrollo para todos ellos. Iberdrola hace presente ese compromiso

\footnotetext{
47 Bolewski, W. (2020).

48 Egea, M. A.; Parra-Meroño, M. C. y Wandosell, G. (2020).

49 Ruël, H. (2020).
} 
en su Politica corporativa de relación con los grupos de interés ${ }^{50}$, mediante el denominado Universo de Relaciones Iberdrola en el que interactúan todos esos grupos.

Figura 2. Plano de relaciones de la empresa con sus grupos de interés

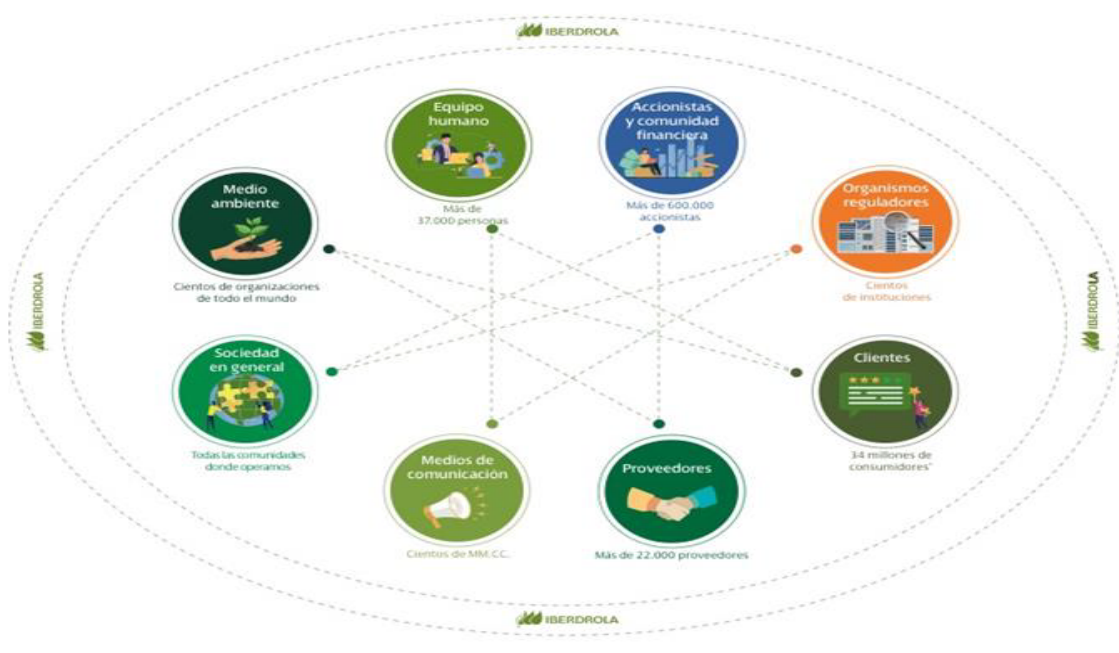

Fuente: https://www.iberdrola.com/conocenos/energetica-del-futuro/grupos-de-interes

Aunque no olvidemos que pueden existir tantos tipos de diplomacia corporativa como tipos de empresa. Por lo tanto, en el caso de Iberdrola, podríamos afirmar que el modelo de diplomacia corporativa se basa esencialmente en tres ejes: las relaciones institucionales, la comunicación corporativa y la reputación. Asimismo, se pone de manifiesto que Iberdrola ha enfocado la gestión acorde con el tipo de empresa y sus objetivos más allá de las fronteras de su país. Así, Iberdrola se ha configurado como un actor destacado en uno o

50 Así denominada en su página web. La empresa, desde la anterior crisis y su salida al exterior, es muy consciente del impacto social de sus decisiones y cuida con especial interés la relación con tales grupos; permanece atenta a las corrientes de opinión y procura mantener un diálogo inclusivo con todos ellos para evitar confrontación al tratarse de un producto sometido a la opinión pública; es también sensible a la protección y a la ayuda de los grupos vulnerables, no solo en España, sino sobre todo con los países en los que colabora. 
más países, debido no solo a la consecución de importantes inversiones directas extranjeras, sino a ser capaces de influir en la opinión pública, esencialmente.

\section{CONCLUSIONES}

De cuantos conceptos y referencias se han puesto de relieve en este artículo, se extraen las siguientes conclusiones, en consonancia con los objetivos que habíamos planteado al principio.

La diplomacia corporativa (DC), aun siendo un concepto acuñado en el siglo XXI, se inspira para sus acciones y procedimientos en la diplomacia pública, puesto que, como ella, se encamina a su primer objetivo, captar la mejor y más eficaz información, a veces, de forma más rápida, sutil y operativa, lo que le permite conocer las corrientes de opinión que subyacen en el mercado para prevenir y anticiparse. Las funciones de la diplomacia pública son, de este modo, una útil matriz para el ejercicio empresarial; se ha visto en el caso de la información y otro tanto puede aseverarse de la representación llevada a cabo por los delegados o country manager, particularmente atentos a la palpitación social y económica del país, y encargados de tejer las mejores relaciones y contactos.

Por otro lado, la acción diplomática oficial deja un campo extenso al ejercicio empresarial y le sirve de modelo para su acción exterior; esta, a su vez, continúa el camino emprendido históricamente por las asociaciones comerciales, las compañías y los embajadores informales en la búsqueda de representación y actividad que procure presencia aceptada, relaciones con el ámbito público, influencia y buena reputación. Además, a la hora de precisar los rasgos que deben reunir quienes se dediquen profesionalmente al ejercicio diplomático corporativo, los definidos por los clásicos para sus legados, embajadores y cortesanos son un punto de partida valioso y contrastado por su eficacia. En este sentido, las empresas necesitan los mejores «embajadores» de su marca que apliquen técnicas de DC para proyectar su identidad corporativa antes sus grupos de interés en el ámbito internacional. La consecución de buena imagen y buena reputación es uno de los cometidos más apreciados para las empresas y que mejor define la política exterior corporativa. De hecho, las empresas que encomiendan a sus departamentos de RSC y de DC la búsqueda de necesidades entre sus públicos de interés, como el caso de Iberdrola, suelen recibir el aprecio y la valoración social. 
Por lo tanto, las empresas que aplican los principios de la DC han conseguido visibilidad en el complicado panorama económico, además de reconocimiento a su labor (Iberdrola); sin embargo, se ha comprobado que hasta ahora solo las grandes empresas aplican estos principios, salvo las empresas nacidas con vocación internacional y vinculación desde origen al mercado exterior, y son partidarias de mantener departamentos o expertos en esa línea. Sería aconsejable que en las empresas medianas y pequeñas también penetrase el concepto de los beneficios que resultan de abordar el mercado desde una vertiente más relacional y aplicar esos recursos, bien en forma de consultoría o bien en forma de asociación entre empresas que necesitan lograr reputación e influencia.

Se desprende del estudio la toma de conciencia de la gran empresa respecto a las ventajas de ejercer influencia beneficiosa para su marca y, también, la necesidad de integrar paulatinamente a la pequeña y mediana empresa en esta persuasiva y eficaz estrategia no comercial de relaciones e interés por lo genuino del país de acogida muy productiva, sin embargo, para cimentar la buena reputación de la empresa que brinda la diplomacia corporativa. Con este objetivo, organismos, instituciones y asociaciones empresariales deben asumir una tarea de divulgación imprescindible.

Por último, de lo investigado se deduce la necesidad de formar sobre este perfil profesional en la universidad y las escuelas de negocios mediante estudios específicos y prácticas eficaces. Algunas escuelas ${ }^{51}$, universidades y organismos ya han creado másteres que ofrecen esa especialidad o ponen la base para que la desarrollen más tarde como carrera profesional. Estos programas ${ }^{52}$ dotan de las herramientas necesarias para la toma de decisiones, la gestión de intereses, la gestión de la influencia y la comprensión del posicionamiento reputacional de las empresas en el complejo contexto mundial en el que, por otra parte, se impone la progresiva transformación digital ${ }^{53}$.

51 http://iidc.es/actividades/formacion/programas-abiertos/

52 https://www.ucam.edu/estudios/cursos/diplomacia-corporativa-inteligencia-representacion-de-interes

53 https://nuevotempocomunicacion.com/blog/la-diplomacia-corporativa-5-0-en-los-nuevos-tiempos/ 


\section{BIBLIOGRAFÍA}

Asquer, Alberto (2012), "What is Corporate Diplomacy?", SSRN Electronic fournal, DO- 10.2139/ssrn.2009812

Barciela, Fernando (2010), "Diplomacia en clave corporativa", El País https://elpais.com/diario/2010/03/07/negocio/1267970611_850215.html

Bolewski, W. (2021) "Compass for Public/Private Management in Turbulent Times: Corporate Diplomacy" International fournal of Diplomacy and Economy, vol. 7, nº 1, p. 13.

Cabarcos, Rafael y S. Ponz, Carlos (2017), Diplomacia Corporativa: Una nueva herramienta de gestión directiva para generar valor a través del capital relacional. Global Corporation Center. Fundación EY e IE Bussines School, https://docplayer.es/51184063-Diplomacia-corporativa.html

Camuñas, Antonio (2012), "Los nuevos conceptos de poder: La diplomacia corporativa en España", Retos de nuestra acción exterior: Diplomacia pública y Marca España, n 18, pp. 107-118.

Carrió-Invernizzi, Diana (2015), "Los artistas y la diplomacia”, en Imágenes del poder en la Edad Moderna, Editorial Universitaria Ramón Areces, Madrid, pp. 77-89.

Convenio de Viena sobre Relaciones Diplomáticas (18 de abril de 1961) https://www.boe.es/boe/dias/1968/01/24/pdfs/A01031-01036.pdf

Earley, P. Christopher y Ang, Soon (2003), Cultural Intelligence: An Analysis of Individual Interactions Across Cultures, University Press, Stanford.

Egea Medrano, Manuel A. (2016), Poder e influencia para operar en mercados internacionales: la Diplomacia Corporativa como herramienta de dirección estratégica. Tesis doctoral. Universidad Católica de Murcia, España.

Egea Medrano, Manuel A.; Parra-Meroño, María Concepción y Wandosell, Gonzalo (2020), "Corporate Diplomacy Strategy and Instruments; With a Discussion about "Corporate Diplomacy and Cyclical Dynamics of Open Innovation", Fournal of Open Innovation: Technology, Market, and Complexity, vol. 6, nº 3. https://doi.org/10.3390/joitmc6030055

Fanjul, Enrique (2010), "Factores culturales e internacionalización de la empresa y la economía", Revista ICE, nº 856, pp. 7-20. 
Fanjul, Enrique (2013), "La diplomacia comercial y la internacionalización de la economía y la empresa", $A R I, \mathrm{n}^{\circ} 11$ del Real Instituto Elcano, https://www.files.ethz.ch/isn/164418/ARI11-2013-Fanjul-diplomacia-comercial-internacionalizacion-empresa-economia.pdf

Fernández Carrasco, Eulogio (2012), "Guerra y diplomacia en la Edad Moderna”, Revista de Derecho UNED, n 10, pp. 183-207.

Freeman, Robert E. [1984 (2010)], Strategic Management: A Stakebolder Approach, Pitman Publishing Inc., Boston.

Freeman, Robert E.; Harrison, Jeffrey S.; Wicks, Andrew C.; Parmar, Bidhan L. y De Colle, Simone (2010), Stakeholder Theory: The State of the Art, Cambridge University Press, Cambridge.

Gombrich, Ernst H. (2014), La bistoria del arte, Phaidon Press, Nueva York.

Hall, Edward T. (1990), Understanding Cultural Differences, Intercultural Press, Yaremouth.

Harris, Jared D. y Freeman, Robert Edward (2008), "The Impossibility of the Separation Thesis", Business Ethics Quarterly, vol. 18, n 4, pp. 541-548.

Henisz, Witold J. (2014), Corporate Diplomacy. In Building Reputations and Relationships with External Stakeholders; Greenleaf, Sheffield, UK.

Henisz, Witold J. (2016), "The Dynamic Capability of Corporate Diplomacy”, Global Strategy fournal, vol. 6, nº 3, pp. 183-196

Hofstede, Gert; Hofstede Gert Jan; Minkov, Michael (2017), Lokales Denken, Globales Handeln. Verlag C.H. Beck oHG., Munich.

Iberdrola (2017), “Compañía española con mejores prácticas de gobierno corporativo, según World Finance"” (24 de abril), El Economista, https://www.eleconomista.es/economia/noticias/8312226/04/17/Iberdrolacompania-espanola-con-mejores-practicas-de-gobierno-corporativo-segunWorld-Finance.html

Iberdrola (2020), Política de relaciones con los grupos de interés, (15 de diciembre). https://www.iberdrola.com/wcorp/gc/prod/es_ES/corporativos /docs/politica_relaciones_grupos_interes.pdf

López Amores, Antonio (2014), "El giro copernicano de la diplomacia: un enfoque desde la tratadística del Renacimiento", Fòrum de Recerca, ${ }^{\circ}{ }^{\circ}$ 19, pp. 89-106. 
López Estrada, Francisco (2005), "Ruy González de Clavijo. La embajada a Tamerlán. Relato del viaje hasta Samarcanda y regreso (1403-1406)", Arbor CLXXX, pp. 711-712. DOI: 10.3989/arbor.2005.1711/712.456

Martín Romero, José Julio (2016), "La Batalla campal de los perros contra los lobos", Una fábula moral de Alfonso de Palencia", Libros de la Corte, $\mathrm{n}^{\circ} 12$, pp. 111-155.

Maillo-Pozo, Rubén (2014), "El humanismo cívico en Castilla a mediados del siglo XV: la Batalla campal de los perros contra los lobos de Alfonso de Palencia", CUNY Academic Works, https://academicworks.cuny.edu /gc_etds/450

Manfredi Sánchez, Juan Luis (2018), Diplomacia corporativa. La nueva inteligencia directiva, Editorial UOC, Barcelona.

Mattingly, Garrett (1970), La Diplomacia del Renacimiento, Instituto de Estudios Políticos, Madrid.

Mogensen, Kirsten (2020), "Legitimacy Issues in Corporate Public Diplomacy”, en Rendtorff, Jakob Dahl (Ed.), Handbook of Business Legitimacy: Responsibility, Ethics and Society, Springer VS, Cham, pp. 1277-1293.

Ochoa Brun, Miguel Ángel (2002), Embajadas y embajadores en la Historia de España, Aguilar, Madrid.

Quintana, Manuel José [1984 (2010)], Cartas a Lord Holland sobre los sucesos políticos de España en la segunda época constitucional, Ediciones Alfar, Sevilla.

Revilla Canora, Javier (2015), "Puedo hacer de una plumada cuantos duques quiera, pero ni un solo Rubens. Un artista diplomático al servicio de la Casa de Austria", Revista de Historia Moderna, no 33, pp. 101-122.

Romero, Isidoro y Rodríguez-Gutiérrez, M. José (2014), "Sobre la internacionalización de la pyme y su contribución a la imagen exterior de España", Comillas Fournal of International Relations, $\mathrm{n}^{\circ}$ 1, pp. 91-104.

Ruël, Huub (2020), "Multinational Corporations as Diplomatic Actors: An Exploration of the Concept of Business Diplomacy", Diplomatica, vol. 2, $\mathrm{n}^{\circ} 1$, pp. 1-12.

Sarfati, Gilberto (2007), Manual de Diplomacia Corporativa. A Construção das Relações Internacionais da Empresa, Grupo Editorial Atlas, Sâo Paulo.

Sevin, Efe (2021), "The Missing Link: Cities and Soft Power of Nations", International fournal of Diplomacy and Economy, vol. 7, nº 1, pp. 19-32 
Shell, Richard G. (2005), Bargaining for Advantage: Negotiation Strategies for Reasonable People, Viking Penguin, Nueva York.

Torres Corominas, Eduardo (2010), "El Cortesano de Castiglione: modelo antropológico y contexto de recepción en la corte de Carlos V", Centros de poder italianos en la monarquía bispánica (siglos XV-XVIII). Actas del Congreso, vol. 2, pp. 1183-1234. https://www.academia.edu/17203672/El_cortesano_de_Castiglione_modelo_antropol\%C3\%B3gico_y_contexto_de_recepci\%C3\%B3n_en_la_Corte_de_Carlos_V

Villarreal Larrinaga, Oskar y Landeta Rodríguez, Jon (2010), “El estudio de casos como metodología de investigación científica en dirección y economía de la empresa. Una aplicación a la internacionalización” Investigaciones Europeas de Dirección y Economía de la Empresa, vol. 16, nº 3, pp. 31-52. 

RECENSIONES 
